\title{
Cerebellopontine angle meningiomas: postoperative outcomes in a modern cohort
}

\author{
Vijay Agarwal, M.D., ${ }^{1}$ Ranjith Babu, M.S., ${ }^{1}$ Jordan Grier, B.S., ${ }^{1}$ \\ Owoicho Adogwa, M.D., M.P.H., ${ }^{1}$ Adam Back, M.D. ${ }^{1}$ Allan H. Friedman, M.D. ${ }^{1}$ \\ Takanori Fukushima, M.D., ${ }^{1}$ and Cory Adamson, M.D., Ph.D., M.P.H., M.H.Sc. ${ }^{1-3}$ \\ ${ }^{1}$ Division of Neurosurgery, Department of Surgery, and ${ }^{2}$ Department of Neurobiology, Duke University \\ Medical Center; and ${ }^{3}$ Neurosurgery Section, Durham VA Medical Center, Durham, North Carolina
}

\begin{abstract}
Object. Tumors of the cerebellopontine angle (CPA) have always proven difficult for neurosurgeons to optimally manage. Studies investigating the natural history and treatment of vestibular schwannomas have dominated the literature in this regard. Distinguishing meningiomas from schwannomas in this location carries particular importance as each tumor type has certain prognostic and surgical considerations. In this study, the authors have characterized the outcomes of 34 patients surgically treated for CPA meningiomas and have investigated various factors that may affect postoperative neurological function.

Methods. The medical records of patients with CPA meningiomas who underwent surgery from 2005 to 2013 at the Duke University Health System were reviewed. Various patient, clinical, and tumor data were gathered from the medical records including patient demographics, pre- and postoperative neurological examinations, duration of symptoms, procedural details, tumor pathology and size, and treatment characteristics. Differences in continuous variables were then analyzed using the Student t-test while categorical variables were evaluated using the chi-square test.

Results. A total of 34 patients underwent surgical treatment for CPA meningiomas during the 8-year period. Jugular foramen invasion was seen in $17.6 \%$ of tumors, with nearly half $(41.2 \%)$ extending into the internal acoustic canal. The most common presenting symptom was hearing loss $(58.8 \%)$, followed by headache $(52.9 \%)$ and facial numbness/pain $(50.0 \%)$. The most common cranial nerve $(\mathrm{CN})$ affected was CN X (11.8\%), followed by CNs VI and VII (5.9\%). Postoperatively, no patients experienced a decrease in hearing, with only $5.9 \%$ of patients experiencing facial nerve palsies. Patients with tumors larger than $3 \mathrm{~cm}$ had a significantly higher incidence of permanent $\mathrm{CN}$ deficits than those with smaller tumors $(45.5 \%$ vs $5.9 \%$, respectively; $\mathrm{p}=0.011)$. Also, tumor extension into the jugular foramen was associated with the occurrence of lower $\mathrm{CN}$ deficits, none of which occurred in tumors without jugular foramen invasion. Internal acoustic canal tumor extension was not seen to be associated with postoperative complications or $\mathrm{CN}$ deficits.

Conclusions. Meningiomas of the CPA are challenging lesions to treat surgically. However, the risk of facial palsy and hearing loss is significantly lower when compared with vestibular schwannomas. Novel methods for preoperative differentiation are needed to appropriately counsel patients on surgical risks. Also, due to the significant potential for neurological deficits, further studies are needed to investigate the utility of radiotherapy for these lesions. (http://thejns.org/doi/abs/10.3171/2013.10.FOCUS13367)
\end{abstract}

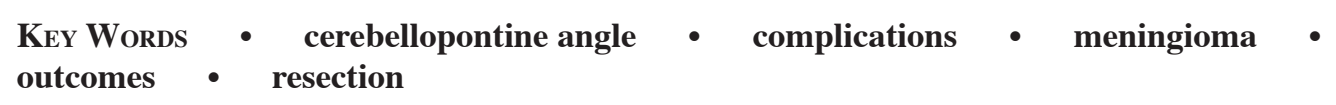

$\mathrm{O}$ NE of every 10 intracranial tumors originates in the cerebellopontine angle (CPA), most of which are schwannomas and meningiomas. ${ }^{36}$ Meningiomas are typically benign tumors that originate from the cells of the arachnoid villi, while the former is a benign tumor that most commonly stems from Schwann cells of the vestibular nerve sheath. Meningiomas in this region present a particularly significant challenge to surgically treat due to the ample blood supply, points of neural at-

\footnotetext{
Abbreviations used in this paper: $\mathrm{CN}=$ cranial nerve; $\mathrm{CPA}=$ cerebellopontine angle; GTR = gross-total resection; IAC = internal acoustic canal; STR = subtotal resection; VS = vestibular schwannoma.
}

tachment, and compression of eloquent structures including the brainstem. ${ }^{20,34,37}$ Distinguishing meningiomas from schwannomas in this location carries particular importance, as each tumor type has certain prognostic and surgical considerations. Special anatomical considerations include involvement of the internal acoustic canal (IAC) and jugular foramen, involvement of bony structures, cranial nerve $(\mathrm{CN})$ involvement, and possible brainstem invasion. Important treatment considerations in this location include the preservation of vestibulocochlear and facial nerve function as tumors in this location often adhere to these nerves. Previous studies have typically consisted of small cohorts treated over long periods and have demonstrated variable surgical outcomes that 
are primarily dependent on tumor characteristics. ${ }^{17,20}$ One such study of 24 patients demonstrated that $46 \%$ experienced short-term complications, with $92 \%$ of lower $\mathrm{CN}$ neuropathies occurring in patients with tumors extending into the jugular foramen. ${ }^{17}$ In this current study, we have characterized the outcomes of 34 patients with CPA meningiomas surgically treated for over an 8-year period and have investigated various factors that may affect postoperative neurological function.

\section{Methods}

\section{Patient Cohort}

This study was approved by the institutional review board at Duke University. The medical records of patients with CPA meningiomas that were surgically treated from 2005 to 2013 at the Duke University Health System were reviewed. The preoperative imaging studies of all patients were examined by 2 independent reviewers to confirm the primary location in the CPA. Patients were excluded if they had undergone previous surgical management or radiotherapy.

\section{Surgical Treatment}

The clinical status and preoperative MR images of all patients were reviewed to determine the most appropriate surgical strategy. Based on surgeon preference, patients with large tumors underwent preoperative embolization in which polyvinyl alcohol particles were used if the supplying vessels were accessible. Surgeries involved use of advanced microsurgical techniques and intraoperative navigation systems, as well as neuromonitoring and CSF drainage.

\section{Data Collection}

Various patient, clinical, and tumor data were gathered from the medical records including patient demographics, pre- and postoperative neurological examinations, duration of symptoms, procedural details, tumor pathology and size, radiological findings, and treatment characteristics. Permanent complications were characterized as those that persisted for 6 months or longer postoperatively. Magnetic resonance images were evaluated to determine the extent of resection, presence of recurrence, and lesion size. Gross-total resection (GTR) was defined as the complete absence of the lesion on postoperative MRI. The presence of a recurrent lesion was evaluated using postoperative MRI and defined as the radiological progression of the tumor. Additionally, imaging studies and operative reports were used to determine tumor extension into the IAC and jugular foramen.

\section{Statistical Analysis}

The data were summarized using medians for continuous variables, and counts and percentages for categorical variables. Differences in continuous variables were analyzed using the Student t-test, while categorical variables were evaluated using the chi-square test. Values with $\mathrm{p}<0.05$ were considered statistically significant. All analyses were performed using SAS 9.3 (SAS Institute).

\section{Results \\ Patient and Tumor Characteristics}

A total of 34 patients underwent surgical treatment for CPA meningiomas during the 8-year period. The median age was 57.5 years (range 20-80 years), with the majority of patients being female (79.4\%) (Table 1). Most tumors (91.2\%) were WHO Grade I, with the remaining being WHO Grade II lesions. While the majority of WHO Grade I tumors were of unspecified subtype, known tumor subtypes included secretory $(n=3)$, fibroblastic $(n=2)$, psammomatous $(n=1)$, microcystic $(n=$ $1)$, meningothelial $(n=1)$, and angiomatous $(n=1)$. Jugular foramen invasion was seen in $17.6 \%$ of tumors, with nearly half (41.2\%) extending into the IAC (Figs. 1 and 2). The median tumor size was $2.3 \mathrm{~cm}$ (range $0.9-4.6 \mathrm{~cm}$ ).

\section{Radiological Presentation}

In 2 cases (5.9\% [ 2 of 34]), a clear operative description was lacking and/or MR images on record were insufficient for determination of tumor attachment, and in 8 cases preoperative CT scans were absent (used to determine the presence of tumor calcification). Tumor attachment was classified as tentorial (3 [8.8\%] of 34 patients), anterior petrous (anterior to meatus, 11 [32.4\%] of 34 patients), posterior petrous (posterior to meatus, 11 [32.4\%] of 34 patients), superior to meatus (3 [8.8\%] of 34 patients), or petroclival (4 [11.8\%] of 34 patients). A clas-

TABLE 1: Characteristics of 34 patients with CPA meningiomas treated at a single institution from 2005 to $2013^{*}$

\begin{tabular}{cc}
\hline Characteristic & Value \\
\hline patient related & \\
age in yrs & 57.5 \\
median & $20-80$ \\
range & \\
sex & $7(20.6)$ \\
male & $27(79.4)$ \\
female & \\
race & $23(67.6)$ \\
Caucasian & $9(26.5)$ \\
black & $2(5.9)$ \\
other & \\
tumor related & \\
WHO grade & $31(91.2)$ \\
I & $3(8.8)$ \\
II & \\
tumor extension & $6(17.6)$ \\
jugular foramen & $14(41.2)$ \\
IAC & \\
size in cm & 2.3 \\
median & $0.9-4.6$ \\
range &
\end{tabular}

* Values are presented as the number of patients (\%) unless otherwise indicated. 


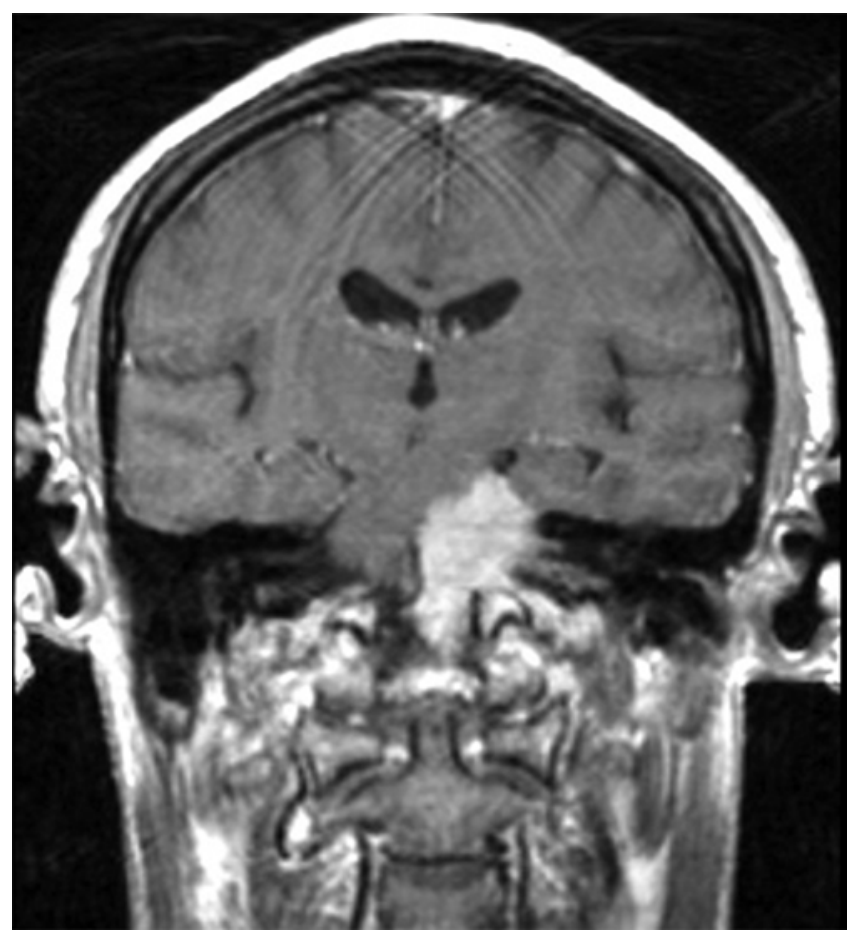

FIG. 1. Preoperative Gd-enhanced MR image demonstrating an enhancing CPA mass extending into the jugular foramen.

sic broad-based, or sessile, appearance was documented in $23(71.9 \%)$ of 32 patients, whereas a rounded appearance was shown in the remaining cases. Hyperostosis was present in $12(37.5 \%)$ of 32 patients, as determined from preoperative CT scans and/or anteroposterior/lateral skull radiographs. None of the tumors in this series exhibited calcification, while a dural tail was found in 23 patients $(71.9 \%)$. Tumor attachment site, the presence of hyperostosis, or the presence of a dural tail did not significantly affect postoperative outcome.

\section{Clinical Presentation}

The median duration of symptoms was 7 months (range 0.1-120 months) (Table 2). The most common presenting symptom was hearing loss (58.8\%); this was followed by headache $(52.9 \%)$ and facial numbness/pain $(50.0 \%)$. Other presenting symptoms included ataxia, double vision, tinnitus, dysarthria, dysphagia, lower-ex-
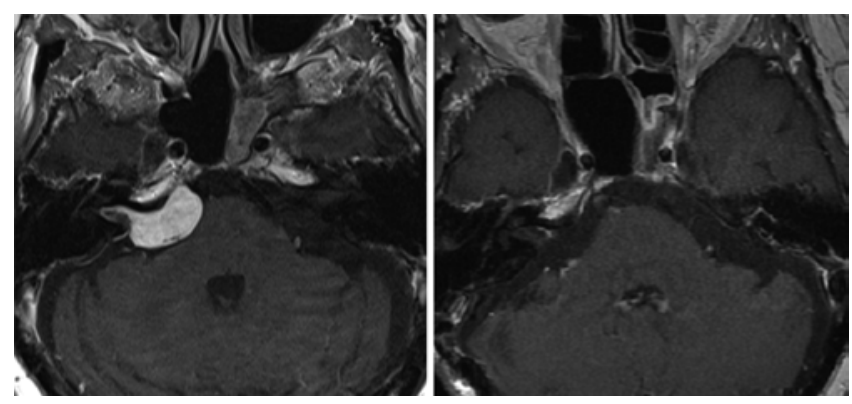

FIG. 2. Left: Preoperative Gd-enhanced MR image demonstrating an actively enhancing mass at the right CPA extending into the IAC. Right: Postoperative MR image demonstrating residual linear enhancement in the IAC to preserve facial nerve function.
TABLE 2: Presenting characteristics in the 34 patients with CPA meningiomas*

\begin{tabular}{lc}
\hline \multicolumn{1}{c}{ Characteristic } & Value \\
\hline symptoms & \\
hearing loss & $20(58.8)$ \\
headache & $18(52.9)$ \\
facial numbness/pain & $17(50.0)$ \\
ataxia & $14(41.2)$ \\
double vision & $6(17.6)$ \\
tinnitus & $5(14.7)$ \\
dysarthria & $5(14.7)$ \\
dysphagia & $4(11.8)$ \\
tongue numbness & $3(8.8)$ \\
facial weakness & $2(5.9)$ \\
weakness & $1(2.9)$ \\
signs & \\
CN neuropathy & $25(73.5)$ \\
duration of symptoms in mos & 7 \\
median & $0.1-120$ \\
range &
\end{tabular}

* Values are presented as the number of patients (\%) unless otherwise indicated.

tremity weakness, tongue numbness, and facial weakness. Evaluation of the effect of IAC tumor extension on clinical presentation did not reveal a significant difference in the incidence of preoperative hearing loss between patients with and without IAC tumor extension (64.3\% vs $52.6 \%$, respectively; $\mathrm{p}=0.52$ ).

\section{Treatment}

Preoperative embolization was attempted in 4 patients (11.8\%). However, embolization was unsuccessful in 3 patients due to an inability to access feeding vessels. Successful embolization in the 1 case occurred via branches of the right internal maxillary, right accessory meningeal, right middle meningeal, and right ascending pharyngeal artery. A number of different surgical approaches were used for the resection of CPA meningiomas (Table 3). The most commonly used was the retrosigmoid approach $(35.3 \%)$. Other approaches included the retromastoid $(23.5 \%)$, combined retrolabyrinthine-retrosigmoid (17.6\%), translabyrinthine (11.8\%), transcondylar $(8.8 \%)$, and retrolabyrinthine $(2.9 \%)$ approaches. Grosstotal resection was achieved in $55.9 \%$ of patients (Fig. 3). Examination of the effect of tumor size on the extent of resection revealed that GTR-treated patients had significantly smaller tumors than subtotal resection (STR)treated patients ( 2.0 vs $4.1 \mathrm{~cm}$, respectively; $\mathrm{p}<0.0001)$. Seven patients $(20.6 \%)$ underwent postoperative radiotherapy, all of whom received STR. The tumors typically received 2500-5400 cGy in fractions of 180-500 cGy. All WHO Grade II meningiomas received postoperative radiation treatment. Only 1 patient with a WHO Grade II meningioma underwent chemotherapy (bevacizumab). 
TABLE 3: Treatment and outcome characteristics in the 34 patients with CPA meningiomas*

\begin{tabular}{cc}
\hline Characteristic & No. of Patients (\%) \\
\hline treatment related & \\
approach & \\
retrosigmoid & $12(35.3)$ \\
retromastoid & $8(23.5)$ \\
combined approach & $6(17.6)$ \\
translabyrinthine & $4(11.8)$ \\
transcondylar & $3(8.8)$ \\
retrolabyrinthine & $1(2.9)$ \\
extent of resection & \\
GTR & $19(55.9)$ \\
STR & $15(44.1)$ \\
postoperative treatment & \\
chemotherapy & $1(2.9)$ \\
radiotherapy & $7(20.6)$ \\
complication related & \\
new CN deficits & $12(35.3)$ \\
CN III & $1(2.9)$ \\
CN IV & $1(2.9)$ \\
CN V & $1(2.9)$ \\
CN VI & $2(5.9)$ \\
CN VII & $2(5.9)$ \\
CN VIII & $0(0.0)$ \\
CN IX & $0(0.0)$ \\
CN X & $4(11.8)$ \\
CN XI & $1(2.9)$ \\
CN XII & $0(0.0)$ \\
permanent CN deficits & $8(23.5)$ \\
temporary CN deficits & $5(14.7)$ \\
wound infection & $2(5.9)$ \\
CSF leak & $2(5.9)$ \\
tracheostomy & $2(5.9)$ \\
PEG tube placement & $2(5.9)$ \\
hematoma & $1(2.9)$ \\
tongue swelling & \\
worsened CN deficits & $1(2.9)$ \\
\hline
\end{tabular}

* PEG = percutaneous endoscopic gastrostomy.

\section{Postoperative Outcomes}

Complications primarily consisted of $\mathrm{CN}$ deficits, occurring in $35.3 \%$ of patients (Table 3). The majority $(66.7 \%)$ of these complications were permanent, accounting for $23.5 \%$ of all patients. Only 1 patient experienced a worsening of a $\mathrm{CN}$ deficit that existed preoperatively. The most common nerve affected was CN X (11.8\%); this was followed by CNs VI and VII (5.9\%). Other complications included wound infection, CSF leaks, tracheostomy requirement, percutaneous endoscopic gastrostomy (PEG) tube placement, hematoma, tongue swelling due to

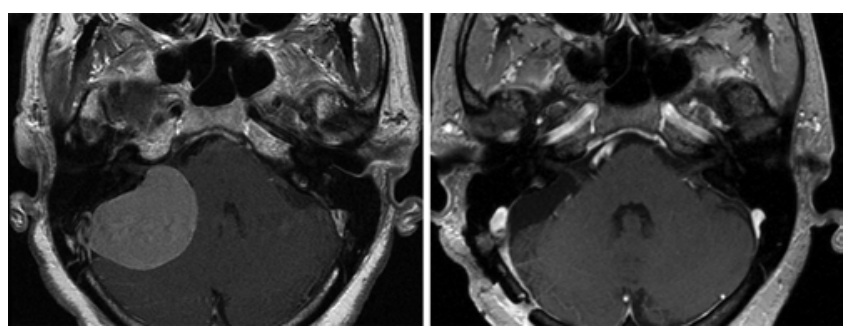

FIG. 3. Left: Preoperative Gd-enhanced MR image demonstrating a large enhancing mass at the right CPA resulting in mass effect on the brainstem, middle cerebellar peduncle, and cerebellum. Right: Postoperative MR image demonstrating GTR of the mass.

traumatic intubation, and dizziness. Of note, no patients experienced a decrease in hearing postoperatively.

Various factors, such as tumor size/extension and the extent of resection, were found to affect the incidence of complications. Patients with tumors larger than $3 \mathrm{~cm}$ had a significantly higher incidence of permanent $\mathrm{CN}$ deficits than those with smaller tumors $(45.5 \%$ vs $5.9 \%$, respectively; $\mathrm{p}=0.011)$. Also, tumor extension into the jugular foramen was associated with the occurrence of lower $\mathrm{CN}$ deficits, none of which occurred in tumors without jugular foramen invasion. Four patients experienced $\mathrm{CN}$ $\mathrm{X}$ deficits, one of whom required gastrostomy tube placement for long-term enteral access. One patient also experienced a CN XI palsy, resulting in left trapezius weakness. All lower CN deficits (CNs IX-XII) were seen to be permanent. In contrast, IAC tumor extension was not found to be associated with postoperative complications or CN deficits. Finally, STR was shown to significantly increase the rates of permanent $\mathrm{CN}$ deficits compared with GTR (40.0\% vs $10.5 \%$, respectively; $p=0.046)$.

At a mean follow-up duration of 16.6 months (range 0-50.2 months), 3 patients (8.8\%) had tumor progression, 2 of whom had WHO Grade II tumors. Progression occurred at 2.6, 28.8, and 45.0 months following primary resection. All patients with tumor progression underwent STR during the primary procedure.

\section{Discussion}

Tumors of the CPA have always proven difficult for neurosurgeons to manage optimally. They are often intimately involved with CNs and various blood vessels, posing great risk to surgical intervention. Factors such as pathology, tumor size and location, extent of resection, and preoperative $\mathrm{CN}$ function have been implicated in long-term outcome. ${ }^{5,17,25,28,32,35,37}$ Studies investigating the natural history and treatment of vestibular schwannomas (VSs) have dominated the literature in this regard. ${ }^{2-4,6-16,21,23,26,29-31,38}$ However, as meningiomas account for $10 \%-15 \%$ of CPA tumors, it is also important to examine the postoperative outcomes following the resection of these tumors as well. Furthermore, due to the prognostic effect of tumor pathology on postoperative neurological outcomes, differentiating these tumors from VSs by examining imaging and clinical features is increasingly important. Nonetheless, few studies have examined the factors affecting the surgical outcome of CPA meningio- 
mas. In the present study we have examined the postoperative complications of a large modern cohort of patients with CPA meningiomas treated at a single institution to investigate various preoperative and perioperative factors that may affect postsurgical outcome.

Well described are the radiographic differences between meningiomas and schwannomas in this location. ${ }^{19}$ The literature indicates that meningiomas of the CPA are more broad-based/sessile with dural tails, ${ }^{18,19}$ and our study found similar imaging results, with broad-based or sessile appearance in $78.1 \%$ and a dural tail $71.9 \%$. One of the distinguishing radiological differences between meningiomas and VSs is the presence of hyperostosis, found in up to approximately $70 \%$ of CPA meningiomas. ${ }^{19}$ Our study found this characteristic in just $37.5 \%$ of patients. Another feature with a strong influence on preoperative evaluation is tumor attachment site. This factor takes on an even greater importance because tumors in this difficult location may influence the surgical approach itself. Kunii et al. retrospectively classified CPA meningiomas in 34 patients into 4 types: 1$)$ tentorial type $(n=13)$, with attachment to the dura mater from the tentorium to the anterior petrous bone, and trigeminal nerve displaced caudally or medially; 2) petroclival type $(n=7)$, with attachment medial to the trigeminal nerve, which is usually displaced laterally; 3 ) anterior petrous type $(n=6)$, with attachment to the dura mater of the anterior petrous bone or Meckel's cave, and trigeminal nerve displaced rostrally or medially; and 4) posterior petrous type $(n=8)$, with attachment posterior to the internal auditory meatus. ${ }^{18}$ This study borrowed from Al-Mefty, ${ }^{1}$ with the addition of the tentorial subtype, who classified posterior fossa meningiomas into 6 subtypes: petroclival, sphenopetroclival, clival, foramen magnum, anterior petrosal, and posterior petrosal. In our study, similar to that of Kunii et al., we found a comparable number of anterior and posterior petrous attachments (32.4\% and $32.4 \%$, respectively). However, the number of tentorial and petroclival attachments in our study was substantially lower (8.8\% and $11.8 \%$, respectively). In addition, due to tumor configuration and intraoperative findings, we found the need for an additional category to maintain descriptively accurate results: superior meatal attachments (3 [8.8\%] of 34 patients). As mentioned, the tumor attachment site did not significantly affect surgical outcome.

Tumors of the CPA have traditionally been associated with high rates of postoperative facial palsy and/or hearing loss because resection poses a particularly challenging endeavor. Systematic reviews of outcomes following VS resection have revealed that $22 \%$ of patients have postoperative complications, with the rates of facial nerve palsy ranging from $8 \%$ to $25 \% .{ }^{34}$ Additionally, the surgical management of VSs has been shown to result in poor rates of serviceable hearing, with prospective studies demonstrating hearing preservation rates ranging from $0 \%$ to $5 \% . .^{24,27}$ To determine the comparative postsurgical outcomes following the resection of VS and CPA meningiomas, single-institutional series are needed to allow for direct comparisons. Recently, Nonaka et al. reported the complications following the surgical management of VS at our institution from 2000 to $2009 .{ }^{26}$ The main neurological complication was facial palsy, which was observed in $14 \%$ of cases. Other neurological complications included disequilibrium (6.3\%), facial numbness $(2.2 \%)$, and lower $\mathrm{CN}$ deficit $(0.5 \%)$. Hearing preservation was attempted in 170 patients $(41.5 \%)$ with small to medium tumors $(<2 \mathrm{~cm})$, with useful hearing preserved in 129 cases $(75.9 \%)$. Gross-total resection was achieved in $88.2 \%$ of these 170 patients, with hearing preservation in $75.3 \%$ (compared with $82.4 \%$ and $66.7 \%$ in cases of near-total and subtotal resection, respectively). Hearing preservation surgery was also attempted in 29 patients with tumors $21 \mathrm{~mm}$ and greater, with a success rate of $82.8 \%$ (24 patients).

In contrast, the current study has demonstrated very low rates of facial palsy and no cases of hearing loss postoperatively. Two patients $(5.9 \%)$ presented after surgery with new hemifacial palsy (both permanent). The substantially lower rates of facial nerve palsy and hearing loss, found to be $5.9 \%$ and $0 \%$, respectively, for CPA meningiomas in our study, is likely attributed to a more intimate relationship between VS and CNs VII and VIII as these typically invade the IAC and cause significant nerve compression. ${ }^{6,7}$ Vestibular schwannomas tend to be more intimately involved with CN VIII as they originate along the axon of this nerve from the glial-Schwann sheath junction up to termination within auditory or vestibular end organs. Meningiomas, however, do not arise from the nerve and therefore can more easily be peeled away with less or no injury to the nerve complex. However, in both VS and meningiomas of the CPA, care should be taken with large or giant lesions, with special thought toward leaving a residual capsule before a decrease in facial nerve response or auditory brainstem response occurs.22 Also of great importance to neurosurgeons to improve outcomes is choosing a reasonable operative approach that maximizes their comfort and familiarity with the anatomy and potential complications. The most common complication seen in the current study was dysphagia due to lower CN dysfunction (11.8\%). Previous studies examining CPA meningiomas have also demonstrated similar outcomes..$^{17,31}$ A study by Kane et al., which examined 24 patients treated over a 16-year period, revealed that $17 \%$ and $33 \%$ of patients had CN IX and X neuropathies, respectively. ${ }^{17}$ The vast majority (92\%) of these complications involved resection of CPA meningiomas that extended into the jugular foramen. In a study by Sanna et al., similar results were shown, with new postoperative lower $\mathrm{CN}$ dysfunction occurring in $62 \%$ of patients with tumors extending into the jugular foramen. ${ }^{31}$ In our study, all 4 patients with such palsies had tumor extension into the jugular foramen. In contrast, VS resection is associated with lower rates of lower $\mathrm{CN}$ palsies, with data from our institution revealing $0.5 \%$ of patients experiencing such a deficit. ${ }^{26}$ This is likely due to the lower rates of jugular foramen invasion by VS tumors.

To mitigate the risks of deficits such as facial palsy and hearing loss, there has been increasing interest in the use of radiotherapy for lesions in the CPA, particular with VS. Radiotherapy has been found to be associated with a decreased incidence of temporary facial weakness compared with microsurgery ( $4.8 \%$ vs $14 \%$, respectively), as well as a significantly higher quality of life at 5-year follow-up. ${ }^{39}$ While few prospective studies have been performed, most 
have consistently shown a decreased incidence of postoperative facial nerve dysfunction and hearing loss in cases involving small to medium VSs. ${ }^{24,27,40}$ However, data for radiotherapy in cases of CPA meningiomas are sparse. One study by Starke et al. evaluated outcomes in patients with skull base meningiomas treated with Gamma Knife surgery (GKS), both as an adjunct to microsurgery and as a primary treatment. ${ }^{33}$ Analysis of data in 255 patients (43 with CPA meningiomas) showed that, at a median of 6.5 years after surgery, tumor volume either did not change or decreased in 220 patients $(86 \%)$. Similarly, a total of 230 patients $(90 \%)$ showed improvement or no change in neurological condition. In our study, 7 patients who underwent STR received radiotherapy postoperatively. Due to the promise of radiotherapy for the management of VS, further studies are needed to investigate its utility for CPA meningiomas. Also, with the heightened risk associated with large invasive tumors of the CPA, as demonstrated above, and the potential benefits of focused radiotherapy on meningiomas in this location, patients in whom the jugular foramen is clearly involved may benefit from an aggressive resection primarily focused on the noninvasive portion of tumor. This strategy is supported by the low incidence of hearing loss in our series.

Large meningiomas $(>3 \mathrm{~cm})$ of the CPA pose an even greater technical challenge and are associated with a high rate of complications..$^{20}$ In our study, permanent $\mathrm{CN}$ complications were found in $45.5 \%$ of the cases in which lesions were greater than $3 \mathrm{~cm}$ compared a rate of $5.9 \%$ in patients with lesions less than $3 \mathrm{~cm}(\mathrm{p}=0.011)$. In a study of CPA meningiomas larger than $3 \mathrm{~cm}$, Leonetti et al. used a combined retrosigmoid-transpetrosal-transcochlear approach and found postoperative complications in 8 patients $(27.6 \%) .{ }^{20}$ These included 7 cases of new $\mathrm{CN}$ deficits (3 CN VII, 2 CN VI, and 1 each of CN V and X weakness) and 1 case of temporary CSF leakage. In general, larger lesions tend to have a more tenuous blood supply and intricate neural attachments. ${ }^{20}$ It was noted that the extended approach allowed for GTR in 19 (66\%) of 29 patients. In our study, GTR was achieved in $19(55.9 \%)$ of 34 patients, although it was only achieved in $9.1 \%$ of patients with tumors larger than $3 \mathrm{~cm}$. Permanent $\mathrm{CN}$ deficits postoperatively were found to be significantly higher in those who underwent STR compared with GTR (40.0\% vs $10.5 \%$, respectively; $p$ $=0.046)$. This was due to larger tumor size as the average size of tumors with GTR was $2.0 \mathrm{~cm}$ compared with 4.1 $\mathrm{cm}$ for those with STR.

For those patients with significantly decreased hearing, often expressed as a tone audiogram threshold below $50 \mathrm{~dB}$ and speech discrimination scores of less than $50 \%$, approaches such as the translabyrinthine and transcochlear are used. ${ }^{20}$ However, the retrosigmoid approach is commonly employed when attempts at hearing preservation are a priority. This approach was thus used most commonly $(35.3 \%)$, allowing for our low rate of hearing loss. Other approaches used included retromastoid $(n=8)$, translabyrinthine $(n=4)$, transcondylar $(n=3)$, retrolabyrinthine $(n=1)$, and a combined approach (retrolabyrinthine/retrosigmoid; $n=6$ ). The type of approach was not significantly related to outcome or complications. In general, the approach chosen should depend on maximal exposure for the particular tumor location, surgeon familiarity, and patient preference to allow for optimal outcome.

\section{Conclusions}

Meningiomas of the CPA are challenging lesions to treat surgically. Most CPA meningiomas are Grade I and can be completely resected via a retrosigmoid approach with excellent progression-free survival. Preoperative embolization should be considered for larger tumors. Intraoperative neuromonitoring of brainstem auditory evoked potentials and involved CNs should be employed. Grade I CPA meningiomas that are subtotally resected due to intimate involvement of CNs should be observed, whereas postoperative radiation therapy should be considered for Grade II CPA meningiomas. Patients may develop CN deficits postoperatively, with complication risk increasing with larger tumor size and tumor extension into the jugular foramen. However, the risk of facial palsy and hearing loss is significantly lower compared with VSs. In the present study, only $5.9 \%$ of patients experienced facial nerve palsies, with no patients having a decrease in hearing function postoperatively. Due to the similar appearance of CPA meningiomas and VSs on imaging, novel methods for preoperative differentiation are needed to appropriately counsel patients on surgical risks. Also because of the significant potential for neurological deficits, further studies are needed to investigate the utility of radiotherapy for these lesions.

\section{Disclosure}

The authors report no conflict of interest concerning the materials or methods used in this study or the findings specified in this paper.

Author contributions to the study and manuscript preparation include the following. Conception and design: Agarwal, Babu, Fukushima. Acquisition of data: Agarwal, Babu, Grier, Back, Fukushima. Analysis and interpretation of data: Agarwal, Babu, Fukushima. Drafting the article: Agarwal, Babu, Adogwa. Critically revising the article: Agarwal, Babu. Reviewed submitted version of manuscript: Adamson, Agarwal, Babu, Grier, Adogwa, Back, Friedman. Approved the final version of the manuscript on behalf of all authors: Adamson. Statistical analysis: Babu. Administrative/technical/material support: Adamson. Study supervision: Adamson, Friedman, Fukushima.

\section{References}

1. Al-Mefty O: Operative Atlas of Meningiomas. Philadelphia: Lippincott-Raven, 1998

2. Arriaga MA, Chen DA, Fukushima T: Individualizing hearing preservation in acoustic neuroma surgery. Laryngoscope 107:1043-1047, 1997

3. Arts HA, Telian SA, El-Kashlan H, Thompson BG: Hearing preservation and facial nerve outcomes in vestibular schwannoma surgery: results using the middle cranial fossa approach. Otol Neurotol 27:234-241, 2006

4. Babu R, Sharma R, Bagley JH, Hatef J, Friedman AH, Adamson C: Vestibular schwannomas in the modern era: epidemiology, treatment trends, and disparities in management. Clinical article. J Neurosurg 119:121-130, 2013

5. Bassiouni H, Hunold A, Asgari S, Stolke D: Meningiomas of the posterior petrous bone: functional outcome after microsurgery. J Neurosurg 100:1014-1024, 2004 
6. Bennett M, Haynes DS: Surgical approaches and complications in the removal of vestibular schwannomas. Neurosurg Clin $\mathbf{N}$ Am 19:331-343, 2008

7. Briggs RJ, Fabinyi G, Kaye AH: Current management of acoustic neuromas: review of surgical approaches and outcomes. J Clin Neurosci 7:521-526, 2000

8. Charpiot A, Tringali S, Zaouche S, Ferber-Viart C, Dubreuil C: Perioperative complications after translabyrinthine removal of large or giant vestibular schwannoma: outcomes for 123 patients. Acta Otolaryngol 130:1249-1255, 2010

9. Darrouzet V, Martel J, Enée V, Bébéar JP, Guérin J: Vestibular schwannoma surgery outcomes: our multidisciplinary experience in 400 cases over 17 years. Laryngoscope 114:681-688, 2004

10. Darwish BS, Bird PA, Goodisson DW, Bonkowski JA, MacFarlane MR: Facial nerve function and hearing preservation after retrosigmoid excision of vestibular schwannoma: Christchurch Hospital experience with 97 patients. ANZ J Surg 75: 893-896, 2005

11. Duke DA, Lynch JJ, Harner SG, Faust RJ, Ebersold MJ: Venous air embolism in sitting and supine patients undergoing vestibular schwannoma resection. Neurosurgery 42:1282-1287, 1998

12. Enée V, Guérin J, Bébéar JP, Darrouzet V: [Acoustic neuroma surgery. Results and complications in 348 cases.] Rev Laryngol Otol Rhinol (Bord) 124:45-52, $2003(\mathrm{Fr})$

13. Gantz BJ, Parnes LS, Harker LA, McCabe BF: Middle cranial fossa acoustic neuroma excision: results and complications. Ann Otol Rhinol Laryngol 95:454-459, 1986

14. Gormley WB, Sekhar LN, Wright DC, Kamerer D, Schessel D: Acoustic neuromas: results of current surgical management. Neurosurgery 41:50-60, 1997

15. Isaacson B, Telian SA, El-Kashlan HK: Facial nerve outcomes in middle cranial fossa vs translabyrinthine approaches. Otolaryngol Head Neck Surg 133:906-910, 2005

16. Jain VK, Mehrotra N, Sahu RN, Behari S, Banerji D, Chhabra DK: Surgery of vestibular schwannomas: an institutional experience. Neurol India 53:41-45, 2005

17. Kane AJ, Sughrue ME, Rutkowski MJ, Berger MS, McDermott MW, Parsa AT: Clinical and surgical considerations for cerebellopontine angle meningiomas. J Clin Neurosci 18: 755-759, 2011

18. Kunii N, Ota T, Kin T, Kamada K, Morita A, Kawahara N, et al: Angiographic classification of tumor attachment of meningiomas at the cerebellopontine angle. World Neurosurg 75: 114-121, 2011

19. Kutz JW, Barnett SL, Hatanpaa KJ, Mendelsohn DB: Concurrent vestibular schwannoma and meningioma mimicking a single cerebellopontine angle tumor. Skull Base 19:443-446, 2009

20. Leonetti JP, Anderson DE, Marzo SJ, Origitano TC, Schuman $\mathrm{R}$ : Combined transtemporal access for large $(>3 \mathrm{~cm})$ meningiomas of the cerebellopontine angle. Otolaryngol Head Neck Surg 134:949-952, 2006

21. Mangham CA: Complications of translabyrinthine vs. suboccipital approach for acoustic tumor surgery. Otolaryngol Head Neck Surg 99:396-400, 1988

22. Martin TP, Fox H, Ho EC, Holder R, Walsh R, Irving RM: Facial nerve outcomes in functional vestibular schwannoma surgery: less than total tumour excision significantly improves results. J Laryngol Otol 126:120-124, 2012

23. Moriyama T, Fukushima T, Asaoka K, Roche PH, Barrs DM, McElveen JT Jr: Hearing preservation in acoustic neuroma surgery: importance of adhesion between the cochlear nerve and the tumor. J Neurosurg 97:337-340, 2002

24. Myrseth E, Møller P, Pedersen PH, Lund-Johansen M: Vestibular schwannoma: surgery or gamma knife radiosurgery? A prospective, nonrandomized study. Neurosurgery 64:654663, 2009

25. Nakamura M, Roser F, Dormiani M, Matthies C, Vorkapic P,
Samii M: Facial and cochlear nerve function after surgery of cerebellopontine angle meningiomas. Neurosurgery 57:7790, 2005

26. Nonaka Y, Fukushima T, Watanabe K, Friedman AH, Sampson JH, McElveen JT Jr, et al: Contemporary surgical management of vestibular schwannomas: analysis of complications and lessons learned over the past decade. Neurosurgery 72 (2 Suppl Operative):ons103-ons115, 2013

27. Pollock BE, Driscoll CL, Foote RL, Link MJ, Gorman DA, Bauch CD, et al: Patient outcomes after vestibular schwannoma management: a prospective comparison of microsurgical resection and stereotactic radiosurgery. Neurosurgery 59:77-85, 2006

28. Roser F, Nakamura M, Dormiani M, Matthies C, Vorkapic P, Samii M: Meningiomas of the cerebellopontine angle with extension into the internal auditory canal. J Neurosurg 102:1723, 2005

29. Sade B, Mohr G, Dufour JJ: Vascular complications of vestibular schwannoma surgery: a comparison of the suboccipital retrosigmoid and translabyrinthine approaches. J Neurosurg 105:200-204, 2006

30. Sameshima T, Fukushima T, McElveen JT Jr, Friedman AH: Critical assessment of operative approaches for hearing preservation in small acoustic neuroma surgery: retrosigmoid vs middle fossa approach. Neurosurgery 67:640-645, 2010

31. Sanna M, Taibah A, Russo A, Falcioni M, Agarwal M: Perioperative complications in acoustic neuroma (vestibular schwannoma) surgery. Otol Neurotol 25:379-386, 2004

32. Sekhar LN, Jannetta PJ: Cerebellopontine angle meningiomas. Microsurgical excision and follow-up results. J Neurosurg 60:500-505, 1984

33. Starke RM, Williams BJ, Hiles C, Nguyen JH, Elsharkawy MY, Sheehan JP: Gamma Knife surgery for skull base meningiomas. Clinical article. J Neurosurg 116:588-597, 2012

34. Sughrue ME, Yang I, Aranda D, Rutkowski MJ, Fang S, Cheung SW, et al: Beyond audiofacial morbidity after vestibular schwannoma surgery. Clinical article. J Neurosurg 114: 367-374, 2011

35. Thomas NW, King TT: Meningiomas of the cerebellopontine angle. A report of 41 cases. Br J Neurosurg 10:59-68, 1996

36. Tomogane Y, Mori K, Izumoto S, Kaba K, Ishikura R, Ando K, et al: Usefulness of PRESTO magnetic resonance imaging for the differentiation of schwannoma and meningioma in the cerebellopontine angle. Neurol Med Chir (Tokyo) 53:482-489, 2013

37. Voss NF, Vrionis FD, Heilman CB, Robertson JH: Meningiomas of the cerebellopontine angle. Surg Neurol 53:439-447, 2000

38. Wanibuchi M, Fukushima T, McElveen JT Jr, Friedman AH: Hearing preservation in surgery for large vestibular schwannomas. Clinical article. J Neurosurg 111:845-854, 2009

39. Weber DC, Chan AW, Bussiere MR, Harsh GR IV, Ancukiewicz M, Barker FG II, et al: Proton beam radiosurgery for vestibular schwannoma: tumor control and cranial nerve toxicity. Neurosurgery 53:577-588, 2003

40. Whitmore RG, Urban C, Church E, Ruckenstein M, Stein SC, Lee JY: Decision analysis of treatment options for vestibular schwannoma. Clinical article. J Neurosurg 114:400-413, 2011

Manuscript submitted August 15, 2013.

Accepted October 4, 2013.

Please include this information when citing this paper: DOI: 10.3171/2013.10.FOCUS13367.

Address correspondence to: Cory Adamson, M.D., Ph.D., M.P.H., M.H.Sc., Division of Neurosurgery, DUMC Box 2624, Durham, NC 27710. email: cory.adamson@duke.edu. 